\title{
Dexmedetomidine supplementing scalp block for cerebral abscess drainage in a patient with severe pulmonary hypertension
}

\section{Dear Editor,}

A forty-two-year-old, $67 \mathrm{~kg}$ female, with known pulmonary hypertension $(\mathrm{PH})$, secondary to chronic thromboembolic disease, presented to the emergency department (ED) with lethargy, confusion, increasing headache and temperature. The patient was unwell at home for six days. In ED on examination the patient looked unwell, with Glasgow Coma Scale (GCS) 14/15, blood pressure (BP) $80 / 40 \mathrm{mmHg}$, temperature $39.1^{\circ} \mathrm{C}$, breathing 18 breaths minute-1 and had a normal chest examination on auscultation. Past medical history was complicated; spina bifida at birth, ventriculo-peritoneal shunt placed in early days, revised at the age of nine, osteomyelitis, miscarriages and IVC filter placement and pulmonary endarterectomy five years previously for recurrent DVT and PE. The patient was allergic to latex. Two months back echocardiogram showed dilated right heart, impaired right ventricular (RV) function, right atrial (RA) pressure 20, moderate-to-severe tricuspid regurgitation, severe $\mathrm{PH}$ with estimated RVSP (right ventricular systolic pressure) $103 \mathrm{mmHg}$, consistent with the cardiac catheterisation (about four-monthsold pulmonary vascular resistance (PVR) $>12$ woods unit). Current medications included sildenafil, bisoprolol, warfarin and spironolactone. Acute management in ED included fluid challenges for hypotension and broad-spectrum antibiotics. The computed tomography imaging of her head demonstrated a large area of attenuation within the left parietal lobe, moderate mass effect, partial effacement of the left lateral ventricle and midline shift to the right of approximately $5 \mathrm{~mm}$. Subsequent magnetic resonance imaging showed a possible abscess. Urgent surgery was scheduled. On haematological advice, prothrombin complex concentrate 15 units/kg IV and vitamin K 5 mg IV were given, administered as the patient's INR was 2.58. Prior to patient's arrival, theatre was cleared of latex. Monitoring was applied in accordance with Association of Anaesthetists of Great Britain and Ireland (AAGBI) standards. In addition, the left radial artery and left (ultrasound guided) internal jugular vein were cannulated for invasive monitoring. The patient received fentanyl $50 \mathrm{mcg}$ and ketamine in three incremental doses of $10 \mathrm{mg}$ each. Dexmedetomidine infusion was started at $0.5 \mathrm{mcg} \cdot \mathrm{kg}^{-1} \cdot \mathrm{hr}^{-1}$ and a scalp block using $20 \mathrm{ml}$ 0.375\% bupivacaine without adrenaline was performed. Oxygen was delivered by nasal sponge at $4 \mathrm{Lmin}^{-1} \cdot \mathrm{SpO}_{2}$ was maintained within preoperative values (93-94\%), CVP remained 55-68 $\mathrm{mmHg}$ (within preoperative range). The craniotomy and abscess drainage lasted one hour and went uneventfully with good anxiolysis, arousable patient and stable haemodynamics. Purulent material was drained and taken for further investigations including culture sensitivity for further management. The patient was moved to the recovery bay before being returned to the ward.

Pulmonary hypertension is associated with a high risk of morbidity and mortality in the perioperative period making management of such a patient challenging in terms of associated complications including cardiac arrest. ${ }^{1,2}$ Monitored anaesthesia care along with local and regional anaesthesia techniques are considered over general anaesthesia wherever possible. ${ }^{3,4}$ Sedation in a spontaneously breathing patient may lead to respiratory depression, which may adversely affect pulmonary artery pressure secondary to hypercapnea and respiratory acidosis. Use of dexmedetomidine in patients with $\mathrm{PH}$ has produced encouraging results in previous case reports. ${ }^{5,6}$ We used a combined approach with scalp block and sedation in a spontaneously breathing patient, similar to the described technique for awake craniotomies. In a case series of a paediatric population in the catheterisation laboratory, the use of a dexmedetomidine bolus has been demonstrated to not increase PAP/PVR. ${ }^{3}$

Dexmedetomidine was effective in providing sedation and anxiolysis, and the scalp block could provide adequate analgesia for mini-craniotomy and abscess drainage. Low-dose ketamine provided analgesic and sympathomimetic effects.

Dexmedetomidine infusion with scalp block provided adequate surgical conditions without any unfavourable effect on the pulmonary artery pressure. This technique also avoided opioids and other sedatives which could worsen $\mathrm{PH}$. Further studies on dexmedetomidine will be able to establish its safety in procedural sedation in patients with altered cardiopulmonary status. However, regardless of the technique used, close monitoring of respiratory function and haemodynamics by an experienced anaesthetist in the operating theatre, tailored specifically to the patient's physiological status, is mandatory in accordance with AAGBI for delivery of safe anaesthesia.

\section{Dr Satyavir Singhal', Dr Prashant Kumar²* (D, Dr Ryan Sykes',}

${ }^{1}$ Department of Anaesthetics, Royal Victoria Hospital, Belfast, UK ${ }^{2}$ Department of Anaesthesiology, Pt BDS, Post Graduate Institute of Medical Sciences, Rohtak, India

${ }^{*}$ Dr Prashant Kumar was working as Commonwealth Fellow (Neuroanaesthesia and Neuro Critical Care) Department of Anaesthetics, Royal Victoria Hospital, Belfast, UK during the time of the case management

Corresponding author, email: pk.pgims@yahoo.com

Conflict of Interest: None 


\section{Authors' contributions}

SS: conduct of case, review of literature, drafting article, critical revision of manuscript, and final approval. PK: conduct of case, literature search, drafting article, revision, and final approval. RS: conduct of case, collection of record, revising of draft, and final approval.

\section{ORCID}

PKumar (D) https://orcid.org/0000-0002-6460-8563

\section{References}

1. Friesen RH, Williams GD. Anesthetic management of children with pulmonary arterial hypertension. Paediatr Anaesth. 2008;18(3):208-16.

2. Friesen $\mathrm{RH}$, Nichols $\mathrm{CS}$, Twite $\mathrm{MD}$, et al. The hemodynamic response to dexmedetomidine loading dose in children with and without pulmonary hypertension. Anesth Analg. 2013;117:953-9.

3. Nair AS. Dexmedetomidine in pulmonary hypertension: A review. Anaesth, Pain and Intensive Care.2013;17(3):囚279-81.

4. Nathan AT, Marino BS, Hanna B, Nicolson SC. Novel use of dexmedetomidine in patient with pulmonary hypertension. Paediatr Anaesth 2008;18(8):782-4.

5. Bloor BC, Ward DS, Belleville JP, Maze M. Effects of intravenous dexmedetomidine in humans. II. Hemodynamic changes. Anesthesiology. 1992;77(6):1134-42.

6. But AK, Ozgul U, Erdil F, et al. The effects of pre-operative dexmedetomidine infusion on hemodynamics in patients with pulmonary hypertension undergoing mitral valve replacement surgery. Acta Anaesthesiol Scand. 2006;50(10):1207-12. 